\title{
Composição corporal de jovens e idosas eutróficas e com sobrepeso avaliada pelo método óxido de deutério e diferentes equações antropométricas
}

\author{
Evaluation of body composition of eutrophic young \\ women and eutrophic and overweight \\ elderly women using the deuterium \\ oxide method and different \\ anthropometric equations
}

José Ailton Oliveira CARNEIRO'

Karla Helena Coelho VILAÇA ${ }^{2}$

Karina PFRIMER ${ }^{3}$

Eduardo FERRIOLLI ${ }^{3}$

\section{R E S U M O}

\section{Objetivo}

Avaliar o percentual de gordura corporal, a massa gorda e a massa magra de mulheres jovens eutróficas e idosas eutróficas e com sobrepeso/obesas, pelo método antropométrico (equações generalizadas e específicas) e óxido de deutério, e verificar o coeficiente de concordância entre as equações utilizadas com o método óxido de deutério usado como referência.

\section{Métodos}

Participaram do estudo 48 voluntárias (15 jovens eutróficas, 18 idosas eutróficas e 15 idosas com sobrepeso), com idade entre 18 e 75 anos. O peso corporal foi mensurado usando balança digital e a altura, um estadiômetro

\footnotetext{
1 Universidade Estadual do Sudoeste da Bahia, Departamento de Saúde, Programa de Pós-Graduação em Enfermagem e Saúde. R. José Moreira Sobrinho, s/n., Jequiezinho, 45206-190, Jequié, BA, Brasil. Correspondência para/Correspondence to: JAO CARNEIRO.E-mail: <hitoef@yahoo.com.br>.

2 Universidade Católica de Brasília, Pró-Reitoria de Pós-Graduação e Pesquisa, Programa de Pós-Graduação em Gerontologia. Brasília, DF, Brasil.

3 Universidade de São Paulo, Faculdade de Medicina, Programa de Pós-Graduação em Clínica Médica. Ribeirão Preto, SP, Brasil. Artigo baseado na dissertação de JAO CARNEIRO, intitulada "Análise da oscilação postural estática multissegmentar em jovens eutróficas, idosas eutróficas e idosas obesas”. Universidade de São Paulo; 2010.
} 
em barra vertical. A composição corporal foi avaliada por equações antropométricas e pelo método de óxido de deutério $\left({ }^{2} \mathrm{H}_{2} \mathrm{O}\right)$. Para análise dos dados usou-se a estatística descritiva, o teste $t$ Student, coeficiente de concordância de Lin e o gráfico de Bland e Altman.

\section{Resultados}

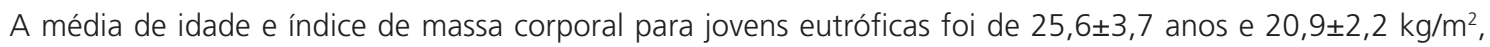
para idosas eutróficas, $69,1 \pm 3,6$ anos e $23,8 \pm 1,8 \mathrm{~kg} / \mathrm{m}^{2}$, e para idosas com sobrepeso/obesas, $69,6 \pm 3,0$ anos e $32,6 \pm 2,6 \mathrm{~kg} / \mathrm{m}^{2}$. Os melhores coeficientes de concordância entre as equações antropométricas e óxido de deutério para a massa gorda foram: jovens, Deutério versus Sloan - 0,70; idosas eutróficas, Deutério versus Jackson - 0,89; e idosas com sobrepeso, Deutério versus Deurenberg - 0,77.

\section{Conclusão}

As equações que apresentaram melhor concordância para massa gorda, massa magra e porcentagem de gordura com o óxido de deutério foram: Sloan para as mulheres jovens; Jackson e Durnin \& Wormersley para as idosas eutróficas e Deurenberg e Visser para as idosas com sobrepeso/obesas.

Palavras-chave: Adulto jovem. Composição corporal. Idoso.

\section{A B S T R A C T}

\section{Objective}

To evaluate the body fat percentage and body lean and fat mass of eutrophic young women and eutrophic and overweight/obese elderly women using anthropometry (generalized and specific equations) and deuterium oxide and to define the concordance coefficient between the equations and the deuterium oxide method (reference).

\section{Methods}

Forty eight volunteers (15 eutrophic young women, 18 eutrophic elderly women, and 15 overweight/obese elderly women), aged between 18 and 75 years, participated in this study. Body weight was measured using a digital scale, and height was measured using a stadiometer with a vertical backboard. Body composition was assessed using anthropometric equations and deuterium oxide $\left({ }^{2} \mathrm{H}_{2} \mathrm{O}\right)$. Data were analyzed using descriptive statistics, Student's t test, Lin's concordance coefficient, and Bland-Altman plot.

\section{Results}

The mean age and body mass index were, respectively: $25.6 \pm 3.7$ years and $20.9 \pm 2.2 \mathrm{~kg} / \mathrm{m}^{2}$ for the eutrophic young women; $69.1 \pm 3.6$ years and $23.8 \pm 1.8 \mathrm{~kg} / \mathrm{m}^{2}$ for the eutrophic elderly women; and $69.6 \pm 3.0$ years and $32.6 \pm 2.6 \mathrm{~kg} / \mathrm{m}^{2}$ for the overweight/obese elderly women. The best concordance coefficients between the anthropometric equations and deuterium oxide for body fat were: eutrophic young women, Deuterium versus Sloan - 0.70; eutrophic elderly women, Deuterium versus Jackson - 0.89; and overweight/obese elderly women, Deuterium versus Deurenberg - 0.77 .

\section{Conclusion}

The equations that showed the best concordance with deuterium oxide were: Sloan for eutrophic young women; Jackson and Durnin \& Wormersley for eutrophic elderly women; and Deurenberg, and Visser for overweight/obese elderly women.

Keywords: Young adult. Body composition. Aged.

\section{N T R O D U Ç Ã O}

O envelhecimento desencadeia modificações na composição corporal do ser humano, observando-se um aumento da massa gorda e uma diminuição da massa livre de gordura'. Atualmente, estima-se que um bilhão de adul- tos esteja com sobrepeso no mundo e cerca de 475 milhões sejam obesos². Uma pesquisa realizada pelo Ministério da Saúde aponta que, no Brasil, esses números chegam a 51 e 17\%, respectivamente ${ }^{3}$, sendo que esse excesso de peso também vem acometendo a população idosa. 
A mensuração acurada da composição corporal em jovens e idosos tem grande relevância na prática clínica e em pesquisas populacionais, tornando-se importante para que programas de intervenções direcionados à adequação entre os compartimentos corporais sejam estabelecidos ${ }^{4,5}$. Para isso, é importante adotar técnicas que sejam precisas para a avaliação da composição corporal, como é o caso do óxido de deutério $\left({ }^{2} \mathrm{H}_{2} \mathrm{O}\right)^{6-8}$. Apesar de ser muito acurada, o óxido de deutério ainda é pouco utilizado na prática clínica e apresenta custos elevados e métodos sofisticados de análise, como o espectrômetro de massa.

As medidas antropométricas usadas como dobras cutâneas e circunferências, podem estimar a gordura subcutânea por meio da avaliação em determinados locais do corpo, além de serem uma medida fácil e menos onerosa. No entanto, algumas dificuldades, como a possível redistribuição da gordura subcutânea, a seleção adequada da equação e a técnica de mensuração, são questões importantes que podem limitar a sua acurácia nos indivíduos idosos ${ }^{1,4}$. Nesse contexto, atualmente um dos grandes desafios dos profissionais da área de saúde que trabalham com composição corporal em adultos e idosos é a escolha da melhor equação antropométrica para identificar a gordura corporal de forma simples, prática, rápida e fidedigna ${ }^{9,10}$.

Devido à importância de se realizar essa análise e à dificuldade em escolher uma equação antropométrica específica para essas populações, este estudo propôs avaliar o percentual de gordura corporal, a massa gorda e a massa magra de mulheres jovens eutróficas e idosas eutróficas e com sobrepeso pelo método antropométrico (equações generalizadas e específicas) e óxido de deutério. Posteriormente foi verificada a concordância entre as equações adotadas, utilizando o método óxido de deutério como referência.

\section{MÉ TO D OS}

Trata-se de um estudo observacional de corte transversal. A amostra foi composta por 48 voluntários do sexo feminino (15 jovens eutróficas, 18 idosas eutróficas e 15 idosas obesas), com idade entre 18 e 75 anos, integrantes do cadastro do Programa de Saúde da Família vinculado à Faculdade de Medicina de Ribeirão Preto da Universidade de São Paulo. As jovens ${ }^{11}$ foram classificadas como eutróficas quando o Índice de Massa Corporal (IMC) apresentou valores entre $18,5-24,9 \mathrm{~kg} / \mathrm{m}^{2}$; já as idosas ${ }^{12}$, quando o IMC esteve $\geq 22$ e $\leq 27 \mathrm{~kg} / \mathrm{m}^{2}$, porém foram classificadas com sobrepeso quando o IMC era $>27 \mathrm{~kg} / \mathrm{m}^{2}$.

Foram excluídas do estudo voluntárias com dificuldades de locomoção, que apresentassem membro amputado, alteração músculo-esquelética, perda localizada de força, sequela de acidente cerebrovascular e que utilizassem órteses ou próteses. O peso corporal foi avaliado por meio de uma balança digital (marca Filizola ID 500, São Paulo [SP], Brasil) com variação de 0,1kg, com o indivíduo vestindo roupas leves e descalço. A estatura corporal foi medida utilizando estadiômetro em barra vertical, inextensível, graduada a cada $0,5 \mathrm{~cm}$.

As pregas cutâneas foram mensuradas pelo mesmo pesquisador com um compasso (marca Lange, Santa Cruz, Califórnia, Estados Unidos) com precisão de 0,1 mm, no hemisfério direito do corpo, em cinco locais: bíceps, tríceps, subescapular, supra-ilíaca e coxa medial, segundo a padronização de Lohman et al. ${ }^{13}$. Foram realizadas três medidas para cada dobra e a média foi usada para análise. A densidade corporal e o percentual de gordura foram obtidos por meio das equações de predição na Tabela 1.

O percentual de gordura corporal foi obtido usando a equação de Siri'19: \%GC=(495/DC)450. A gordura corporal foi calculada multiplicando o Peso Corporal (PC) pelo Percentual de Gordura (\%GC) e dividido por cem (GC=PC $x$ $\% \mathrm{GC} / 100$ ). A massa magra foi obtida subtraindo a Gordura Corporal do Peso Corporal (PC-GC).

Para a avaliação da composição corporal pelo método de óxido de deutério, foi solicitado que as voluntárias permanecessem em jejum por 
um período de oito horas (durante a noite). Depois disso, cada voluntária recebeu $1 \mathrm{~mL} / \mathrm{kg}$ de óxido de deutério (99,9\% óxido de deutério, Cambridge Isotope, Estados Unidos) diluído a 7,0\%, seguido por duas doses de $50 \mathrm{~mL}$ de água natural para a completa ingestão do deutério e para a lavagem da boca. Foram colhidas amostras de saliva antes da ingestão da dose e três horas depois. As amostras foram armazenadas a $-10^{\circ} \mathrm{C}$ até a análise.

O enriquecimento por deutério das amostras de saliva foi determinado por espectrometria de massa de razão isotrópica (Europe Scientific Hydra System, Cheshire, Reino Unido), após equilíbrio com hidrogênio 100\% pelo método do catalisador de platina em alumínio. A composição corporal foi determinada de acordo com o protocolo Schoeller et al. ${ }^{20}$.

Os dados foram apresentados como média \pm desvio-padrão. O teste $t$ Student foi usado para comparar as médias das variáveis de composição corporal avaliadas pelo método óxido de deutério e pelas equações antropométricas. Para verificar a concordância entre as diferentes técnicas usou-se o coeficiente de Lin $^{21}$. Esse coeficiente avalia a reprodutibilidade de medidas, método ou instrumento, podendo variar entre -1 e 1 , e mede o grau de concordância entre os mesmo, sendo que, quanto mais próximo de 1 , mais concordante. Para verificação do grau de concordância utilizou-se a classificação proposta por Landis \& Koch ${ }^{22}$ : sem concordância $(<0,00)$; pobre $(0,00-0,19)$; fraca $(0,20-0,39)$; moderada $(0,40-0,59)$; forte $(0,60-0,79)$; quase perfeita $(0,80-1,00)$. Para as análises descritivas e de associação foi usado o pacote estatístico Statistical Package for the Social Sciences (SPSS Inc., Chicago, Ilinois, Estados Unidos) versão 16.0 e, para as análises de concordância e confecção dos gráficos de Bland e Altman, o pacote estatístico MedCalc versão 9.1.

O estudo foi aprovado pelo Comitê de Ética em Pesquisa do Hospital das Clínicas da Faculdade de Medicina de Ribeirão Preto (Processo $n^{\circ}$ 244/2008). As voluntárias foram informadas dos métodos envolvidos e participaram da pesquisa após assinarem o Termo de Consentimento Livre e Esclarecido.

RES U L T A D OS

Participaram do estudo 48 mulheres (15 jovens, 18 idosas eutróficas e 15 obesas). As características da amostra estão apresentadas na Tabela 2. Na Tabela 3 estão os valores médios do percentual de gordura corporal, massa gorda e massa magra avaliados pelo método óxido de deutério e estimados pelas equações antropométricas generalizadas e específicas para jovens e idosos.

Os coeficientes de concordância da equação de Sloan et al. ${ }^{16}$ versus deutério foram melhores do que os das equações de Jackson et al. ${ }^{14} \mathrm{e}$ Durnin \& Wormersley ${ }^{15}$ no grupo de jovens. Já no de idosas eutróficas os coeficientes de concordância das equações de Jackson et al. ${ }^{14} \mathrm{e}$ Durnin \& Wormersley ${ }^{15}$ foram os melhores, enquanto no grupo de idosas com sobrepeso os coeficientes de concordância das equações de

Tabela 1. Equações antropométricas para a estimativa da densidade corporal e do percentual de gordura em mulheres jovens e idosas.

\begin{tabular}{ll}
\hline Autores & \multicolumn{1}{c}{ Equação } \\
\hline Jackson et al. $1^{14^{*}}$ & $\mathrm{D}=1,0904921-0,0009929\left(\mathrm{X}_{1}\right)+0,0000023\left(\mathrm{X}_{1}\right)^{2}-0,0001392(\mathrm{ID})$ \\
Durnin \& Womersley ${ }^{15^{*}}$ & $\mathrm{D}=1,1339-0,0645\left(\log . \mathrm{X}_{2}\right)$ \\
Sloan et al. ${ }^{16^{* *}}$ & $\mathrm{D}=1,0764-0,00081(\mathrm{SI})-0,00088(\mathrm{TR})$ \\
Visser et al. ${ }^{17^{* * * *}}$ & $\mathrm{D}=0,0226 \times(\mp)-0,0022(\mathrm{IMC})+1,0605$ \\
Deurenberg et al. ${ }^{18^{* * * *}}$ & $\% \mathrm{GC}=1,2(\mathrm{IMC})+0,23(\mathrm{ID})-10,8(\mp)-5,4$ \\
\hline
\end{tabular}

Nota: *Equações generalizadas (jovens e idosos); ${ }^{* *}$ Equação específica (jovem); ${ }^{* * *}$ Equações específicas (idoso). Ғ: Representa a constante para o sexo (feminino=0 e masculino=1). $X_{1}: \sum$ (tricipital, supra-ilíaca e coxa medial); $X_{2}: \sum$ (bicipital, tricipital, subescapular e supra-ilíaca).

SI: Supra-ilíaca; TR: Triciptal; D: Densidade corporal; \%GC: Percentual de Gordura Corporal; ID: Idade (anos); IMC: Índice de Massa Corporal. 
Visser et al. ${ }^{17}$ e Deurenberg et al. ${ }^{18}$ foram os melhores, como apresentado na Tabela 4. Na Figura 1, os gráficos de Bland e Altman confirmam os resultados de concordância do percentual de gordura apresentados na Tabela 4, entre as equações antropométricas e o método deutério nos diferentes grupos.

\section{I S C U S S Ã O}

O presente estudo avaliou e comparou os valores de percentual de gordura corporal, gordura corporal e massa magra de cinco equações antropométricas, sendo uma específica para adultos, duas generalizadas (adultos e idosos) e duas

Tabela 2. Características físicas da amostra estudada. Ribeirão Preto (SP), 2008-2009.

\begin{tabular}{|c|c|c|c|c|c|c|}
\hline \multirow{3}{*}{ Variáveis } & \multicolumn{2}{|c|}{ Jovem } & \multicolumn{4}{|c|}{ Idoso } \\
\hline & \multirow[b]{2}{*}{$\mathrm{M}$} & \multirow[b]{2}{*}{ DP } & \multicolumn{2}{|c|}{ Eutrófico } & \multicolumn{2}{|c|}{ Obeso } \\
\hline & & & M & $\mathrm{DP}$ & M & DP \\
\hline Idade (anos) & 25,6 & 3,7 & 69,1 & 3,6 & 69,6 & 3,0 \\
\hline Peso (kg) & 54,4 & 6,5 & 60,1 & 7,4 & 76,3 & 6,4 \\
\hline Altura (m) & 1,61 & 0,03 & 1,58 & 0,0 & 1,52 & 0,0 \\
\hline $\mathrm{IMC}\left(\mathrm{kg} / \mathrm{m}^{2}\right)$ & 20,9 & 2,2 & 23,8 & 1,8 & 32,6 & 2,6 \\
\hline Tricipital (mm) & 18,9 & 3,7 & 18,6 & 3,5 & 28,9 & 4,1 \\
\hline Bicipital (mm) & 8,7 & 2,6 & 11,0 & 3,5 & 18,7 & 4,4 \\
\hline Supra-ilíaca (mm) & 31,3 & 6,6 & 25,5 & 6,7 & 35,6 & 6,5 \\
\hline Subescapular (mm) & 17,4 & 6,6 & 15,7 & 4,2 & 30,2 & 6,4 \\
\hline Coxa média (mm) & 28,9 & 6,4 & 29,3 & 7,5 & 41,7 & 6,2 \\
\hline
\end{tabular}

Nota: IMC: Índice de Massa Corporal; M: Média; DP: Desvio-Padrão.

Tabela 3. Percentual de gordura, massa gorda e massa magra de jovens e idosos estimados por diferentes métodos. Ribeirão Preto (SP), 2008-2009.

\begin{tabular}{|c|c|c|c|c|c|c|}
\hline \multirow{2}{*}{ Métodos } & \multicolumn{2}{|c|}{$\% G C$} & \multicolumn{2}{|c|}{ Massa gorda $(\mathrm{kg})$} & \multicolumn{2}{|c|}{ Massa magra $(\mathrm{kg})$} \\
\hline & $M$ & $\mathrm{DP}$ & $M$ & $\mathrm{DP}$ & $M$ & $\mathrm{DP}$ \\
\hline \multicolumn{7}{|l|}{ Jovem $(n=15)$} \\
\hline Deutério & 29,7 & $5,4^{\alpha \dagger}$ & 16,4 & $4,8^{\dagger}$ & 38,0 & $2,6^{\dagger}$ \\
\hline Durnin \& Wormersley & 38,6 & 2,6 & 21,1 & 3,7 & 33,2 & 3,0 \\
\hline Jackson & 33,9 & 4,0 & 18,6 & 4,1 & $35,7^{ \pm}$ & 3,3 \\
\hline Sloan & 28,5 & 3,5 & $15,7^{ \pm}$ & 3,5 & 38,7 & 3,7 \\
\hline \multicolumn{7}{|l|}{ Idoso eutrófico $(n=18)$} \\
\hline Deutério & 35,7 & $5,4^{\#^{*}}$ & 21,8 & 5,5 & 38,8 & 3,2 \\
\hline Durnin \& Wormersley & 37,6 & 2,6 & 22,7 & 4,0 & 37,3 & 3,7 \\
\hline Jackson & 34,9 & 4,5 & 21,2 & 5,0 & 38,8 & 3,3 \\
\hline Visser & 41,0 & 1,9 & 24,7 & 4,0 & 35,3 & 3,4 \\
\hline Deurenberg & 39,0 & 2,4 & 23,6 & 4,1 & 36,5 & 3,6 \\
\hline \multicolumn{7}{|l|}{ Idoso obeso $(n=15)$} \\
\hline Deutério & 48,1 & $3,9^{\alpha \dagger}$ & 37,1 & 4,8 & 39,1 & $4,1^{\alpha \dagger}$ \\
\hline Durnin \& Wormersley & 44,2 & 1,9 & 33,8 & 3,6 & 42,5 & 3,3 \\
\hline Jackson & 44,2 & 3,2 & 33,8 & 4,7 & 42,4 & 2,9 \\
\hline Visser & 50,7 & 2,9 & 38,8 & 5,0 & 37,4 & 2,3 \\
\hline Deurenberg & 49,8 & 3,0 & 38,1 & 4,9 & 38,1 & 2,6 \\
\hline
\end{tabular}

Nota: ${ }^{\alpha}$ Diferença significativa entre deutério versus Jackson et al. ${ }^{14 .}{ }^{\dagger}$ Deutério versus Durnin \& Wormersley ${ }^{15}$; \#Deutério versus Visser et al. ${ }^{17}$; *Deutério versus Deurenberg et al. ${ }^{13}$.

\%GC: Percentual de Gordura Corporal; M: Média; DP: Desvio-Padrão. 

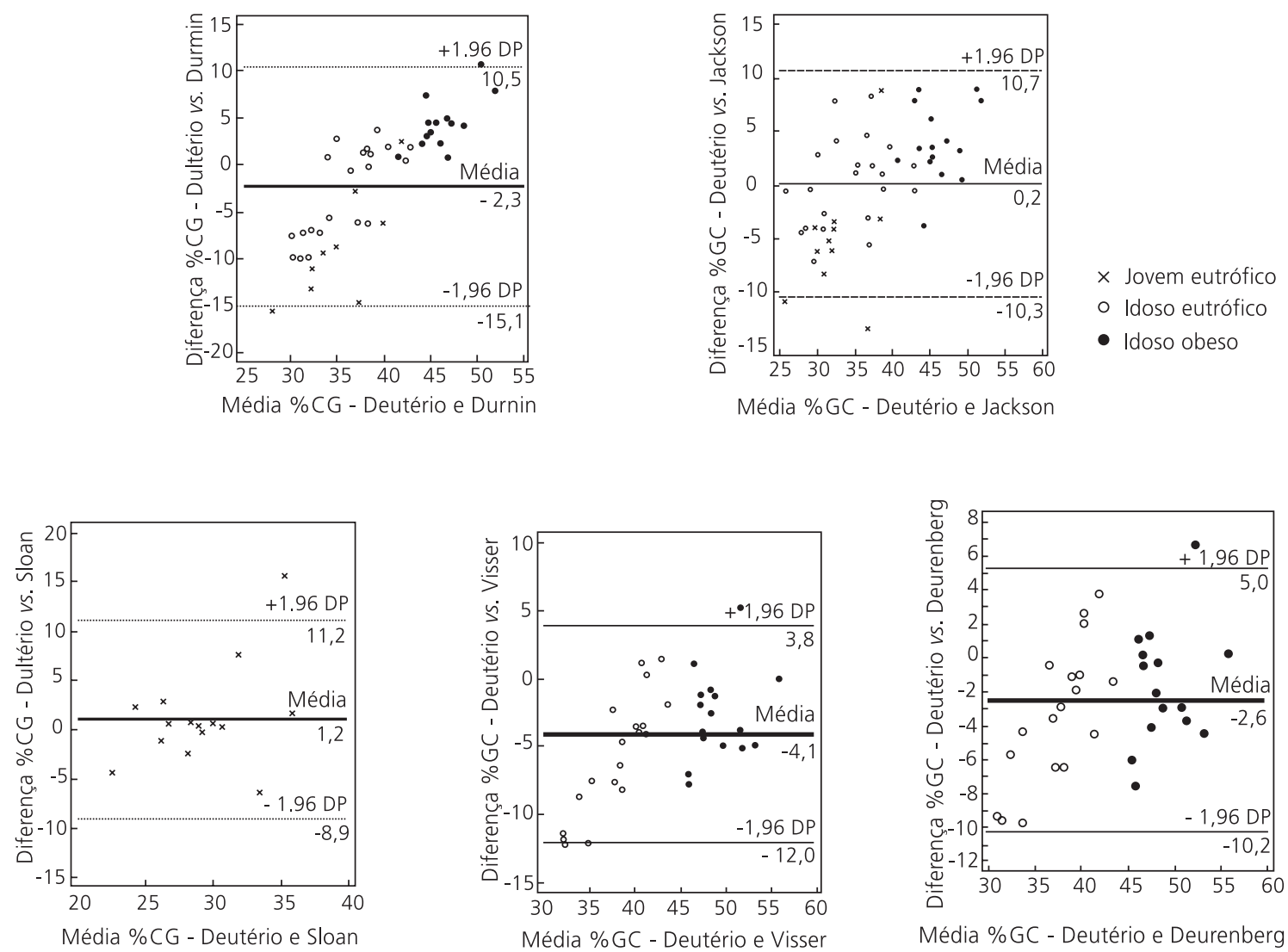

Média \%GC - Deutério e Deurenberg

Figura 1. Gráficos comparando o Percentual de Gordura Corporal (\%GC) entre o método de óxido de deutério e diferentes equações antropométricas. Ribeirão Preto (SP), 2008-2009.

Nota: DP: Desvio-Padrão.

Tabela 4. Coeficiente de concordância entre os métodos deutério e antropométrico. Ribeirão Preto (SP), 2008-2009.

\begin{tabular}{llccc}
\hline \multirow{2}{*}{ Variável } & \multicolumn{1}{c}{ Métodos } & \multicolumn{2}{c}{ Coeficiente de concordância } \\
\cline { 3 - 5 } Percentual de gordura corporal & Deutério versus Durnin & Jovem & Idosa eutrófica & Idosa obesa \\
& Deutério versus Jackson & 0,13 & 0,41 & 0,25 \\
& Deutério versus Sloan & 0,29 & 0,63 & 0,32 \\
& Deutério versus Visser & 0,36 & - & 0,41 \\
& Deutério versus Deurenberg & - & 0,23 & 0,44 \\
Massa gorda $(\mathrm{kg})$ & Deutério versus Durnin & - & 0,39 & 0,59 \\
& Deutério versus Jackson & 0,52 & 0,84 & 0,63 \\
& Deutério versus Sloan & 0,68 & 0,89 & - \\
& Deutério versus Visser & 0,70 & - & 0,75 \\
Massa magra $(\mathrm{kg})$ & Deutério versus Deurenberg & - & 0,73 & 0,77 \\
& Deutério versus Durnin & 0,81 & 0,50 \\
& Deutério versus Jackson & 0,26 & 0,71 & 0,42 \\
& Deutério versus Sloan & 0,38 & 0,72 & - \\
& Deutério versus Visser & 0,50 & - & 0,49 \\
& Deutério versus Deurenberg & - & 0,52 & 0,55 \\
\hline
\end{tabular}


específicas para idosos, com os valores obtidos pelo método óxido de deutério usado como referência. Com base nos achados desta pesquisa, pode-se inferir que a equação de Sloan et al. ${ }^{16}$, específica para adultos, mostrou-se superior às equações generalizadas para avaliar a composição corporal de mulheres jovens, uma vez que os valores de composição corporal apresentaram melhor concordância com o deutério. Também, pode-se inferir que as equações generalizadas de Jackson et al. ${ }^{14}$ e Durnin \& Wormersley ${ }^{15}$ mostraram-se superiores às específicas (Visser et al. ${ }^{17}$, Deurenberg et al. ${ }^{18}$ ) para avaliar a composição corporal de idosas eutróficas. Enquanto as equações específicas para idosos de Visser et al. ${ }^{17} \mathrm{e}$ Deurenberg et al. ${ }^{18}$ mostraram-se superiores às generalizadas (Jackson et al. ${ }^{14}$, Durnin \& Wormersley ${ }^{15}$ ) para avaliar a composição corporal de idosas com sobrepeso, uma vez que os valores de composição corporal apresentaram melhor concordância com o deutério.

No presente estudo, observou-se que os resultados obtidos por meio das equações antropométricas apresentaram concordância de moderada a forte com o deutério para populações distintas. As equações de Jackson et al. ${ }^{14}$ e Durnin \& Wormersley ${ }^{15}$ são generalizadas, ou seja, validadas com jovens e idosos e, por isso, são usadas por muitos profissionais para estimar o percentual de gordura desses indivíduos. No entanto, as equações citadas anteriormente superestimaram os valores de percentual de gordura para mulheres jovens. Sendo assim, torna-se recomendável o uso da equação de Slaon et al. ${ }^{16}$ por ser específica para adultos jovens, apresentando melhor concordância com o método de óxido de deutério.

Entre os idosos eutróficos, as equações generalizadas de Jackson et al. ${ }^{14}$ e Durnin \& Wormersley ${ }^{15}$ apresentaram melhor concordância do que as equações específicas para idosos de Visser et al. ${ }^{17}$ e Deurenberg et al. ${ }^{18}$, as quais superestimaram os valores de gordura corporal. Possivelmente, isso se deve ao fato de as mesmas usarem o valor do IMC em vez do das dobras cutâneas, sendo que o mesmo não é uma estimava confiável para idosos.
Para as idosas com sobrepeso/obesas as equações de Visser et al. ${ }^{17}$ e Deurenberg et al. ${ }^{18}$ apresentaram melhores concordâncias quando comparadas as equações de Jackson et al. ${ }^{14} \mathrm{e}$ Durnin \& Wormersley ${ }^{15}$. Uma possível explicação pode estar relacionada ao fato de que a avaliação da composição corporal por dobras cutâneas não é recomendada para indivíduos com excesso de peso, devido à dificuldade e limitação do adipômetro para pinçar a dobra ${ }^{23}$.

Diferentes estudos observaram uma boa concordância das equações generalizadas de Jackson et al..$^{14}$ e Durnin \& Wormersley ${ }^{15}$ com o método óxido de deutério ${ }^{8,24}$ e com absorciometria radiológica de dupla energia ${ }^{1,25}$. Alguns métodos mais sofisticados para estimar a composição corporal $^{7,26}$, a exemplo da absorciometria por dupla emissão de raios $X$ e do óxido de deutério, muitas vezes são inviáveis para estudo de campo devido ao seu alto custo e da necessidade de pessoas especializadas para sua análise ${ }^{8}$. O método antropométrico ainda é o mais usado pela sua facilidade e economia. No entanto, para o uso dessa técnica também são necessários profissionais treinados para que haja reprodutibilidade e confiabilidade dos dados ${ }^{4,8}$.

Devido à escassez de estudos comparando as diferentes equações antropométricas para estimativa da composição corporal em amostras de idosos estratificada pelo estado nutricional, a comparação com outros estudos foi dificultada. Neste trabalho, as medidas antropométricas foram realizadas por um profissional experiente, $\mathrm{e}$ acredita-se que esse tenha sido um fator importante na obtenção dos resultados concordantes entre os valores estimados pela antropometria e o deutério.

É importante ressalvar que a amostra em questão não foi representativa da população de jovens e idosos por se tratar apenas de indivíduos do sexo feminino; além disso, os métodos utilizados são alguns dos disponíveis para avaliação da composição corporal. Fundamentado nessa afirmação, ressalta-se a necessidade de que mais estudos sobre a avaliação da composição corporal 
em amostras representativas da população de jovens e idosos (homens e mulheres) sejam realizados, para que, dessa forma, possam ser estabelecidos valores de referência que levem em conta as modificações inerentes ao envelhecimento.

\section{O N CLUSÃ O}

Das equações utilizadas nesta pesquisa, a de Sloan et al. ${ }^{16}$ foi a melhor para mulheres jovens eutróficas, as de Jackson et al. ${ }^{14}$ e Durnin \& Wormersley ${ }^{15}$ para as idosas eutróficas e a de Visser et al. ${ }^{17}$ e Deurenberg et al. ${ }^{18}$ para idosas com sobrepeso ao se avaliar a composição corporal quando comparadas ao método óxido de deutério.

\section{COLABORADORES}

JAO CARNEIRO e FERRIOLLI participaram da elaboração e desenho do projeto, análises e interpretação dos dados. KHC VILAÇA e K PFRIMER participaram da elaboração e revisão crítica do artigo.

\section{REFER Ê N CIAS}

1. Barbosa AR, Santarém JM, Filho WJ, Meirelles ES, Marucci MFN. Comparação da gordura corporal de mulheres idosas segundo antropometria, bioimpedância e DEXA. Arch Latinoam Nutr. 2001; 51(1):49-56.

2. International Association for the Study of Obesity. Adult overweight and obesity in the European Union (EU27). London: IASO; 2012 [cited 2013 Nov 2]. Available from: http://www.iaso.org/resources/ world-map-obesity

3. Brasil. Ministério da Saúde. Secretaria de Vigilância em Saúde. Brasil 2012: Vigilância de Fatores de Risco e Proteção para Doenças Crônicas por Inquérito Telefônico. Brasília: Ministério da Saúde; 2013.

4. Aniteli TM, Florindo AA, Pereira RMR, Martini LA. Desenvolvimento de equação para estimativa da gordura corporal de mulheres idosas com osteoporose e osteopenia através da espessura de dobras cutâneas tendo como referência absorciometria por dupla emissão de raios X. Rev Bras Med Esporte. 2006; 12(6):366-70.

5. Rezende F, Rosado L, Franceschinni S, Rosado G, Ribeiro R, Marins JCB. Revisão crítica dos métodos disponíveis para avaliar a composição corporal em grandes estudos populacionais e clínicos. Arch Latinoam Nutr. 2007; 57(4):327-34.

6. Albala C, Yanes M, Salazar G, Vio F. Body composition in the elderly: Total body water and anthropometry. Nutr Res. 1994; 14(12):1797-09.

7. Pfrimer K, Moriguti JC, Lima NKC, Marchini JS, Ferriolli E. Bioelectrical impedance with different equations versus deuterium oxide dilution method for the inference of body composition in healthy older persons. J Nutr Health Aging. 2012; 16(2):124-7.

8. Carneiro JAO, Vilaça KHC, Pfrimer K, Costa NKL, Marchini JS, Moriguti JC, et al. Study of body composition in elderly active women by the anthropometric and deuterium oxide methods. Rev Bras Cineantropom Desempenho Hum. 2012; 14(5):615-23.

9. Rech CR, Santos DL, Silva JCN. Desenvolvimento e validação de equações antropométricas para predição da gordura corporal em mulheres entre 50 e 75 anos de idade. Rev Bras Cineantropom Desempenho Hum. 2006; 8(1):5-13.

10. Guedes DP, Rechenchosky L. Comparação da gordura corporal predita por métodos antropométricos: índice de massa corporal e espessuras de dobras cutâneas. Rev Bras Cineantropom Desempenho Hum. 2008; 10(1):1-7.

11. World Health Organization. Obesity: Preventing and managing the global epidemic. Geneva: WHO; 1998.

12. Lipschitz DA. Screening for nutritional status in the elderly. Prim Care. 1994; 21(1):55-67.

13. Lohman TG, Roche AF, Martorell R. Anthropometric standardization reference manual. Champaign (IL): Human Kinetics; 1988.

14. Jackson AS, Pollock ML, Ward A. Generalized equations for predicting body density of women. Med Sci Sports Exerc. 1980; 12(3):175-82.

15. Durnin JVGA, Womersley J. Body fat assessed from total body density and its estimation from skinfold thickness: Measurements on 481 men and women aged from 16 to 72 years. Br J Nutr. 1974; 32(1):77-92.

16. Sloan AW, Burth JJ, Blyth CS. Estimation of body fat in young women. J Appl Physiol. 1962; 17(6):967-70.

17. Visser M, Heuvel EVD, Deurenberg P. Prediction for the estimation of body composition in the elderly using anthropometric data. Br J Nutr. 1994; 71(6): 823-33.

18. Deurenberg P, Weststrate JA, Seidell JC. Body mass index as a measure of body fatness: Age and sexspecific prediction formulas. Br J Nutr. 1991; 65(2): 105-14. 
19. Siri WE. The gross composition of the body. Adv Biol Med Physics. 1956; 4:239-80.

20. Schoeller DA. Mass spectrometry calculations. J Clin Pharmacol. 1986; 26(6):396-9.

21. Lin, Li A. Concordance correlation coefficient to evaluate reproducibility. Biometrics. 1989; 45(1): 255-68.

22. Landis RJ, Koch GG. The measurement of observer agreement for categorical data. Biometrics. 1977; 33(1):159-74.

23. Madden AM, Smith S. Body composition and morphological assessment of nutritional status in adults: A review of anthropometric variables. J Hum Nutr Diet. 2015. http//:dx.doi.org/10.1111/jhn. 12278
24. Amahon AK, Flynn MG, Iglay HB, Stewart LK, Johnson CA, Mcfarlin BK. Measurement of body composition changes with Weight loss in postmenopausal women: Comparison of methods. J Nutr Health Aging. 2007; 11(3):203-13.

25. Rech CR, Cordeir BA, Petroski EL, Vasconcelos FAG. Estimating body fat from skinfold thicknesses in the elderly. Rev Nutr. 2010; 23(1):17-26. http://dx. doi.org/10.1590/S1415-52732010000100003

26. Davidson LE, Wang J, Thornton JC, Kaleem Z, SilvaPalacios F, Pierson RN, et al. Predicting fat percent by skinfolds in racial groups: Durnin and Womersley revisited. Med Sci Sports Exerc. 2011; 43(3):542-9.

Recebido: janeiro 19, 2015 Versão final: junho 18, 2015 Aprovado: julho 8, 2015 
